СУБКЛИНИЧЕСКИЙ ГИПОТИРЕОЗ И МЕТАБОЛИЧЕСКИЙ СИНДРОМ: ОСНОВАНИЯ ДЛЯ МЕДИКАМЕНТОЗНОГО ВМЕШАТЕЛЬСТВА

\author{
(с Л.А. Руяткина*, Д.С. Руяткин
}

Новосибирский государственный медицинский университет, Новосибирск, Россия

Высокая распространенность метаболического синдрома (МС) и субклинического гипотиреоза (СГипо) создает опасность интегрального кардиометаболического риска (КМР). Разрабатывается концепция о повышении уровней тиреотропного гормона (ТТГ) как компоненте МС с ключевой ролью инсулинорезистентности (ИР). Для выделения групп активного вмешательства анализируются дефиниции СГипо с возрастными и гендерными особенностями. Результаты рандомизированных клинических исследований показали более высокую встречаемость предиабета и сахарного диабета 2 типа (СД2) при СГипо, а также положительные ассоциации аутоиммунного тиреоидита (АИТ) с компонентами MC, особенно у женщин в постменопаузе. Анализируется ассоциация СГипо с системной воспалительной реакцией, что может определять повышение КМР. При этом большая часть дисфункции щитовидной железы (ЩЖ) и компонентов МС ассоциирована с ИР.

Дискутируется целесообразность терапии СГипо левотироксином: для инициации лечения не определены пороговые показатели ТТГ; в пожилом возрасте вследствие снижения потребности в тиреоидных гормонах обсуждается увеличение верхнего референсного диапазона ТТГ; отсутствуют данные о снижении сердечно-сосудистого риска и смертности. Параллельно накоплены данные о снижении уровней ТТГ при явном и субклиническом гипотиреозе у пациентов с МС и СД2 на фоне терапии метформином. Предположено гендерное влияние метформина на активность оси гипоталамус-гипофиз-ЩЖ, раскрыт его новый антигипергликемический механизм действия, включающий активацию АМФ-протеинкиназы (adenosine monophosphate (AMP)-activated protein kinase) в гипофизе.

Полагают, что метформин является перспективным терапевтическим средством не только для пациентов с СД2 и заболеваниями ЩЖ, но также МС и ожирением. Многоплановые возможности метформина, включая коррекцию периферической и центральной ИР и снижение уровней ТТГ при СГипо, подчеркивает комплексный подход к профилактике КМР. Форма метформина с пролонгированным высвобождением имеет ряд преимуществ: лучшая переносимость, большая эффективность в коррекции показателей ИР, липидного и углеводного обменов, дополнительное показание - лечение предиабета, возможность назначения при клиренсе креатинина до 30 мл/мин.

КЛЮЧЕВЫЕ СЛОВА: субклинический гипотиреоз; метаболический синдром; тиреотропный гормон; инсулинорезистентность; кардиометаболический риск, метформин.

\title{
SUBCLINICAL HYPOTHYROIDISM AND METABOLIC SYNDROME: REASONS FOR DRUG INTERVENTION
}

\author{
(c) Lyudmila A. Ruyatkina*, Dmitriy S. Ruyatkin
}

Novosibirsk State Medical University, Novosibirsk, Russia

The high prevalence of metabolic syndrome (MS) and subclinical hypothyroidism (SHypo) creates danger of integral cardio-metabolic risk (CMR). A concept is being developed to increase thyroid-stimulating hormone (TSH) levels as a component of MS with the key role of insulin resistance (IR). To identify groups of active intervention, the definitions of SHypo with age and gender characteristics are analyzed. The results of randomized clinical trials showed a higher incidence of prediabetes and type 2 diabetes mellitus (T2DM) in SHypo, as well as positive associations of autoimmune thyroiditis (AIT) with components of MS, especially in postmenopausal women. The association of SHypo with a systemic inflammatory response is analyzed, which can determine an increase in cardio-metabolic risk. At the same time, most of the thyroid dysfunction and the components of MS are associated with insulin resistance.

The feasibility of SHypo treating with levothyroxine is discussed: the threshold parameters of thyroid-stimulating hormone (TSH) are not determined for initiating treatment; in old age, due to a decrease in the need for thyroid hormones, an increase in the upper reference range of TSH is discussed; there is no evidence of a decrease in cardiovascular risk and mortality. In parallel, data have been accumulated on a decrease in TSH levels in overt and subclinical hypothyroidism in patients with MS and T2DM with metformin therapy. The gender effect of metformin on the activity of the hypothalamus-pituitary-thyroid axis was suggested, its new antihyperglycemic mechanism of action, including the activation of the AMP protein kinase (adenosine monophosphate (AMP) -activated protein kinase) in the pituitary gland, was revealed. 
It is possible that metformin is a promising therapeutic agent not only for patients with type 2 diabetes and thyroid disease, but also for MS and obesity. The multifaceted capabilities of metformin, including the correction of peripheral and central insulin resistance and a decrease in TSH levels in patients with SHypo, emphasizes an integrated approach to the prevention of CMR. The prolonged release form of metformin has several advantages: better tolerance, greater efficiency in the correction of IR, lipid and carbohydrate metabolism, additional indication - treatment of prediabetes, the possibility of prescribing for creatinine clearance up to $30 \mathrm{ml} / \mathrm{min}$.

KEYWORDS: subclinical hypothyroidism; metabolic syndrome; thyroid-stimulating hormone; insulin resistance; cardio-metabolic risk; metformin.

\section{СОДЕРЖАНИЕ ТИРЕОТРОПИНА И МЕТАБОЛИЧЕСКИЙ СИНДРОМ}

Уже много лет активно изучается тесная связь дисфункции щитовидной железы (ЩЖ) с МС [1], особенно с позиций интегрального сердечно-сосудистого риска [2]. В этом плане более точен недавно предложенный термин «кардиометаболический риск» (КМР), под которым понимают повышенную склонность к сердечно-сосудистым заболеваниям (СС3) и сахарному диабету 2 типа (СД2) [3]. Кластер его факторов: абдоминальное ожирение (АО), артериальную гипертонию (АГ), предиабет, повышенные триглицериды и холестерин липопротеинов низкой плотности (ХС-ЛПНП) - определяют как метаболический синдром (МС). По сути, концепция КМР акцентирует прогностическое значение МС с ведущей патогенетической ролью инсулинорезистентности (ИР) [4].

В последние годы появился ряд серьезных исследований, результаты которых отражают связь снижения функции ЩЖ с МС и СД2. В перекрестном исследовании ( $n=132$ 346) уровни тиреотропного гормона (ТТГ) в пределах референсных значений прямо коррелировали с риском компонентов МС, включая ИР [5]. Результаты метаанализа отражают связь субклинического гипотиреоза (СГипо) с более высоким риском развития MC (OP 1,31: 1,08-1,60; p=0,006) при этнических различиях [6]. С этими данными согласуется подобная прямая ассоциация, выявленная при использовании критериев International Diabetes Federation (IDF), но не National Cholesterol Education Program and Adult Treatment Panel III (NCEP-ATP III [7]. В то же время в популяции японских женщин ( $\mathrm{n}=11$ 498) продемонстрирована высокая распространенность СГипо и его тесная связь с рядом показателей МС по критериям NCEP-ATP III [8].

Результаты крупного популяционного проспективного исследования Tehran Thyroid Study показали, что СГипо у пациентов с уровнем ТТГ<10 мМЕ/л может прогнозировать МС с течением времени по мере снижения CT4, значения которого отрицательно коррелируют с ИР и положительно с уровнями АД у мужчин [9]. Также показана тесная связь функции ЩЖ с развитием ожирения, а повышение уровня ТТГ связано с отклонениями метаболического статуса при нормальной массе тела (OP 1,22: 1,01-1,48) [10].

По мере накопления доказательств влияния гормонов ЩЖ на компоненты МС [11] представляется важным уточнение механизмов этих взаимосвязей с их клиническим значением. Рецепторы к тиреоидным гормонам (ТГ) присутствуют в миокарде и сосудах, и даже незначительные изменения концентрации тиреоидных гормонов могут повлиять на сердечно-сосудистую физиологию, увеличив риск сосудистой заболеваемости и смертности на 20-80\% [12]. Очевидно, что чувствительность к инсулину самая высокая, а неблагоприятные влияния ЩЖ на параметры МС самые низкие в условиях эутиреоза [11].

В последние годы активно разрабатывается концепция о повышении уровня ТТГ как компоненте МС. Основания для этой теории - увеличение КМР при отклонении референсных уровней ТТГ в сочетании с параметрами MC [13]. Именно гипотиреоз привлекает особое внимание: влияя на частоту сердечных сокращений и артериальное давление (АД), при склонности к дислипидемии, дисфункции эндотелия (ДЭ), сердечной недостаточности (CH), повышению сердечно-сосудистой жесткости и кардиомегалии [12], он ассоциируется с повышенной заболеваемостью и смертностью от ССЗ [14].

Широко обсуждается влияние СГипо на различные органы и системы. Мнения расходятся в отношении сердечно-сосудистого риска, а также подходов к лечению [15]. Последствия СГипо могут зависеть от продолжительности и степени повышения уровня ТТГ. Возникают вопросы: СГипо повышает риск ССЗ и смертность, ухудшает метаболические параметры, следует его лечить L-тироксином [14]?

Для рассмотрения данной проблемы нами проведен анализ литературы за последние 10 лет по данным базы данных PubMed (MEDLINE) и база данных РИНЦ по указанным ключевым словам. Для MEDLINE: subclinical hypothyroidism; metabolic syndrome; thyroid-stimulating hormone; insulin resistance; cardio-metabolic risk; metformin. Для РИНЦ: субклинический гипотиреоз, метаболический синдром, тиреотропный гормон, инсулинорезистентность, кардиометаболический риск, метформин.

\section{ДЕФИНИЦИИ СУБКЛИНИЧЕСКОГО ГИПОТИРЕОЗА}

СГипо определяют, как повышение ТТГ >4,5 и <20,0 мМЕ/л при свободном тироксине (сT4) в референсном диапазоне $[14,16]$. Частота СГипо варьирует от 4 до 20\% взрослых в зависимости от пола (выше среди женщин), возраста (старше 60 лет), индекса массы тела, расы, курения, потребления йода и других факторов [12, 14]. Частота невыявленного СГипо в Европе, по результатам метаанализа Mendes D. et al. (2019), составила 4,11\% [17]. Tekle H.A. et al. (2018) оценили возраст $\geq 40$ лет как независимый фактор субклинического гипотиреоза [18].

СГипо подразделяют на две категории по уровню ТТГ: мягкий (ТТГ от 4,5 до 10,0 мME /л) и выраженный (ТТГ> 10,0 мМЕ /л) [19]. Razvi S. et al. (2018), классифицируя СГипо как класс 1 (TSH >4,0 или 4,5, но <10,0 мME /л) или класс 2 (ТSH > 10,0 мМЕ/л), подчеркивают отсутствие консенсуса в отношении «нормального» верхнего предела ТТГ, что ведет к противоречию в определении, распространенности и клинической значимости СГипо [12]. 


\section{СУБКЛИНИЧЕСКИЙ ГИПОТИРЕОЗ И КАРДИОМЕТАБОЛИЧЕСКИЙ РИСК}

Связи ЩЖ и сердечно-сосудистой системы, постулированные Polikar R. et al. (1993) [20], были подтверждены дальнейшими исследованиями [21]. Поскольку потенциальные факторы риска ССЗ аналогичны явному гипотиреозу [15], считают, что СГипо является их независимым предиктором, связывая риск с возрастом и повышением уровня ТТГ [22]. Отметим, что большинство долгосрочных последствий гипотиреоза были изучены в контексте именно СГипо, поскольку явный гипотиреоз обычно лечится.

Существуют доказательства, что СГипо может повышать риск СС3, особенно у пациентов с ТТГ> 10,0 мМЕ/л, а также сердечно-сосудистую смертность из-за неблагоприятных воздействий главным образом на АД и липиды. ИР, модулируя различные факторы риска, включая коагуляцию, может потенциально увеличить КМР [14]. СГипо у пациентов с АГ служит фактором риска скрытой неэффективности антигипертензивной терапии, ухудшая профиль амбулаторного АД и увеличивая степень поражения органов-мишеней [23].

Наиболее частой патологией сердца при СГипо является диастолическая дисфункция миокарда (ДДМ) [24]. По результатам 12-летнего Cardiovascular Health Study ( $n=3044$, возраст $\geq 65$ лет) риск СН был повышен у пациентов с СГипо без предшествующего КМР [25]. Показано влияние СГипо на толщину интимы-медии сонной артерии, ДДМ, периферическое сосудистое сопротивление, ДЭ и липидный профиль [16], что подтверждают популяционные исследования, анализ которых приведен в обзорной статье Razvi S. et al. (2018) [12].

Деление СГипо на 2 категории (мягкий и выраженный) обусловлено попыткой более четко выделить группы для активного вмешательства с целью улучшения сердечно-сосудистого прогноза. Так, ТТГ >4,0 расценен как сильный индикатор риска развития атеросклероза и инфаркта миокарда у пожилых женщин [26]; у пациентов с ТТГ >10,0 мМЕ/л отмечен более высокий риск развития СН со сниженной фракцией выброса [27]. При этом аномально высокие уровни ТТГ могут быть связаны с общей более низкой скоростью метаболизма и лучшей выживаемостью у пожилых людей [27] и соответствовать лучшим функциональным результатам после перенесенного инсульта [28].

СГипо связан с целым рядом метаболических отклонений, определяющих его КМР. Так, прямые корреляции ТТГ и ХС-ЛПНП у лиц с эутиреозом и достоверно повышенные уровни PCSK9 при СГипо явились основанием для вывода о регулирующей роли ТТГ в печеночной экспрессии PCSK9, что также способствует более высокому уровню ХС-ЛПНП [29]. Сравнивая две аналогичные по полу и возрасту группы (СГипо и эутиреоз), Ebrahimpour A. et al. (2018) выявили при СГипо на фоне более высоких уровней ХС-ЛПНП значимую прямую корреляцию повышенных HOMA-IR и гомоцистеина. Авторы заключили, что СГипо, вероятно, оказывает существенное влияние на ИР - основной фактор риска СД2, а также гомоцистеин - фактор риска СС3 [30]. Эти данные укладываются в концепцию ИР как ключевого патогенетического звена КМР в контексте СГипо [4].

\section{СУБКЛИНИЧЕСКИЙ ГИПОТИРЕОЗ И МЕТАБОЛИЧЕСКИЙ СИНДРОМ}

Распространенность МС увеличивается после менопаузы; роль сопутствующего СГипо уточняется. У женщин с ожирением при СГипо и эутиреозе показана связь МС с более высокими уровнями ТТГ и интерлейкина-б; их концентрации, как и распространенность МС, были выше при СГипо на фоне аутоиммунного тиреоидита (АИТ) [31]. Авторы предположили роль повышенной продукции провоспалительных цитокинов при АИТ в тесной связи МС и СГипо. Позднее именно у женщин вовлечением ТТГ в провоспалительные процессы объяснили связи высоконормального ТТГ с неблагоприятными исходами, в том числе сердечно-сосудистыми [32].

Выявленные в когорте пациентов с СГипо по сравнению с эутиреоидными более высокие уровни интерлейкина-6 и С-реактивного белка, а также их прямая зависимость от уровня ТТГ ( $\mathrm{R}=0,66 ; 0,59$ - соответственно; $\mathrm{p}<0,05)$ подтверждает версию об ассоциации СГипо с системной воспалительной реакцией - универсальным патологическим процессом, что может определять повышение сердечно-сосудистого риска [33]. Эта гипотеза частично подтверждается связью уровней антител к тиреопероксидазе (АТ-ТПО) с НОМА-IR и C-реактивного белком $(p<0,05)$ у лиц без ожирения [34].

В рамках SPECT-China study ( $\mathrm{n}=9082)$ выявленная положительная ассоциация АИТ с $\mathrm{HbA}_{1 c^{\prime}}$ HOMA-IR, AO, гиперлипидемией и МС, особенно у женщин, позволила заключить, что АИТ может повышать потенциальный КМР даже при референсных уровнях ТТГ [35]. В целом хроническое воспаление при аутоиммунных заболеваниях признано фактором, способствующим атеросклерозу и СС3 [36].

При анализе базы данных NHANES 1999-2002 гг. обнаружено, что доля лиц с нарушенной гликемией натощак была выше при наличии СГипо в сравнении с эутиреозом; риск СД2 при СГипо повышался в 2,29 раза [37]. Метаанализ данных из базы PubMed показал, что частота развития СД2 была в 1,09 раза выше при каждом удвоении мМЕ/л ТТГ (95\% ДИ 1,06-1,12), частота предиабета увеличивалась на 15\% (OP 1,15: 1,04-1,26) у пациентов с ТТГ >5,0 мМЕ /л. МС более распространен при СГипо по сравнению с эутиреоидными лицами (ОР 1,31: 1,08-1,60); в то же время осложнения СД2 более распространены при СГипо, что послужило основанием Latin American Thyroid Society в клинических рекомендациях по гипотиреозу настаивать на активном выявлении гипотиреоза у пациентов с СД2 и МС [38].

По результатам данных большого популяционного исследования ( $n=24$ 765), Chang Y.C. et al. (2019) заключают, что высоконормальный ТТГ является маркером КМР, связанного с АО, ИР, повышенным АД, дислипидемией, гиперурикемией, воспалением и гиперкоагуляцией. При этом большую часть связей дисфункции щЖ и МС опосредует ИР [39]. В результате высоконормальному ТТГ отводят роль нового кардиометаболического маркера.

Таким образом, и в отсутствие явной тиреоидной патологии при МС риск СС3 повышается по мере роста уровней ТТГ даже в пределах референсных значений, тем более при СГипо, что делает целесообразным проведение оценки функции ЩЖ у этих пациентов; особенно уязвимы женщины в постменопаузе. 


\section{ПРОБЛЕМА ТЕРАПИИ СУБКЛИНИЧЕСКОГО ГИПОТИРЕОЗА}

Данные метаанализа рандомизированных клинических исследований (РКИ) ведущих баз данных свидетельствуют об отсутствии улучшения качества жизни на фоне терапии левотироксином среди небеременных взрослых с СГипо [40]. Анализ данных Danish National Patient Register ( $n=628$ 953) показал, что заместительная терапия левотироксином у пациентов с СГипо не способствует более низкой смертности или снижению риска инфаркта миокарда [41]. Отдельный анализ из этой же базы данных пациентов с СГипо с сопутствующими СС3 (n=1192) не выявил связи этой терапии с уменьшением общей смертности, МАСЕ (Major adverse cardiovascular events) или госпитализации [42].

Пороговые уровни ТТГ для начала лечения четко не определены. В отсутствие больших РКИ наиболее оправдан старт терапии СГипо при ТТГ $\geq 10,0$ мМЕ/л [16]. Jasim S. et Gharib H. (2018), обосновывая применение возрастных референсных диапазонов ТТГ с целью исключения ненужного диагноза у пожилых, подчеркивают, что субклинические тиреоидные отклонения могут со временем улучшаться, поэтому рекомендуется тщательный мониторинг функции ЩЖ [43].

Это мнение соотносится с данными Leng O. et Razvi S. (2019) о множестве доказательств неэффективности лечения СГипо с помощью левотироксина, особенно у пожилых, в плане улучшения качества жизни, когнитивных способностей, неясном влиянии на сердечно-сосудистые события и смертность [44]. Поскольку референсный диапазон для ТТГ увеличивается с возрастом, у пациентов старшего возраста с гипотиреозом целесообразно ориентироваться на более высокие цели по ТТГ вследствие снижения потребности в гормонах ЩЖ [44].

\section{НОВЫЕ ВОЗМОЖНОСТИ МЕТФОРМИНА: ГИПОТИРЕОЗ И МЕТАБОЛИЧЕСКИЙ СИНДРОМ}

В параллельно продолжающейся дискуссии о целесообразности терапии СГипо левотироксином удивительный эффект выявлен у метформина, широко используемого препарата в терапии СД2 и МС: уровень ТТГ у пациентов с гипотиреозом на терапии левотироксином снижался в ответ на терапию метформином и вновь повышался при ее прекращении [45]. Влияние на ТТГ было изолированным, без изменения уровней сT4 и сТ3, что подтверждено метаанализом 7 исследований, оценивавших уровни ТТГ у пациентов с СД2 на терапии метформином: показано их снижение как при явном гипотиреозе, так и при СГипо, без изменений при эутиреозе [46].

В популяционном исследовании пациентов с СД2 (59 937 с эутиреозом и 5689 с гипотиреозом) монотерапия метформином была связана с повышенным риском низких уровней ТТГ (<0,4 мМЕ/л) у пациентов с леченым гипотиреозом без подобного влияния при эутиреозе [47]. Этот риск был наиболее высоким (130\%) в период 90-180 дней после начала терапии метформином, однако подавления уровней ТТГ (<0,1 мME/л) не отмечено, как и отрицательных кардиологических эффектов. В ретроспективном клиническом исследовании [48] также выявили связь между терапией метформином и значительно более низкой распространенностью диагностированного гипотиреоза.

В исследованиях с малыми выборками по типу «случай-контроль» результаты противоречивы. В двойном слепом клиническом исследовании 60 пациентов с MC при 2,5<ТТГ<10,0 мМЕ / л (СГипо и высоконормальный диапазон) в результате приема 1000 мг метформина в течение 12 недель выявлено достоверное снижение ТТГ, особенно у пациентов с уровнем >5,0 мME/л и АТ-ТПО(+), независимо от корректировки по полу [49]. Однако в открытом РКИ (ТТГ 5,5-10,0 мМЕ/л) через 6 месяцев приема метформина 1500 мг/день не выявлено достоверных различий в уровнях ТТГ, при этом скорость его нормализации была быстрее у лиц с АТ-ТПО(-) в сравнении с АТ-ТПО(+) при улучшении гликемии и чувствительности к инсулину [50].

Метформин снижал уровни ТТГ у пациентов с нелеченым амиодарон-ассоциированным гипотиреозом, наряду со снижением ИР (по данным HOMA-IR) и уровней пролактина (последний, как известно, участвует в патогенезе ИР). Этот эффект был выявлен только у женщин, что позволяет предположить гендерное влияние метформина на активность оси гипоталамусгипофиз-ЩЖ [51].

Снижение уровней ТТГ вследствие лечения метформином у пациентов с диабетом объясняют усилением действия тиреоидных гормонов на гипофиз и активации аденозин-монофосфат-активируемой протеинкиназы (АМФК), ключевого фермента в многоплановых патологических влияниях ИР. Вследствие этого полагают, что метформин является перспективным терапевтическим средством не только у пациентов с СД2, заболеваниями ЩЖ, но также МС и ожирением [52].

Вопреки прежним представлениям об инсулиннезависимости головного мозга, периферическая ИР тесно связана с ИР центральной нервной системы. Рецепторы инсулина экспрессируются с высокой плотностью в гипоталамусе [53], где воспалительные процессы, митохондриальная дисфункция, стресс эндоплазматического ретикулума тесно связаны с дефектами передачи сигналов инсулина в головном мозге (ИР), что ведет к нейродегенеративным нарушениям. В вентромедиальном ядре гипоталамуса высоко ко-экспрессированы рецепторы к тиреоидным гормонам и АМФК, которая играет ключевую роль в регуляции энергетического гомеостаза как центральной нервной системы, так и периферических органов [54].

Таким образом, в дополнение к многоплановым эффектам метформина [55] раскрыт его новый антигипергликемический механизм действия, включающий активацию АМФК, что продемонстрировано в исследованиях in vitro и in vivo; препарат проникает через гематоэнцефалический барьер и участвует в активации АМФ-протеинкиназы в гипофизе [56]. Это ведет к ингибированию белкового ядерного рецептора LXRa, транскрипционного фактора для проопиомеланокортина. В результате последовательно подавляется образование сначала проопиомеланокортина, затем АКТГ, приводя к снижению уровня кортизола и, как следствие, уменьшению гликемии. Отметим также, что синтез пролактина находится под контролем бета- 
эндорфина, другого производного проопиомеланокортина, секретируемого в эквимолярных концентрациях с кортикотропином. По сути, метформин снижает через центральные механизмы уровни трех гормонов, участвующих в формировании ИР: через производные проопиомеланокортина АКТГ и пролактин, а также ТТГ. Кроме того, способность препарата влиять на АМФК рассматривают как одно из ключевых объяснений его противоопухолевого эффекта, в том числе на раки ЩЖ [57], где высокие уровни ТТГ оказывают стимулирующее влияние; снижение уровней ТТГ в этой ситуации лежит в основе супрессивной терапии.

Предполагается, что метформин, продемонстрировав свою эффективность в ряде заболеваний, тесно ассоциированных с возрастом, модулирует старение и даже рассматривается как потенциальная формула против старения [58]. Подобный комплекс возможностей метформина чрезвычайно актуален в ситуации роста распространенности МС в современном обществе и необходимости профилактики как СД2, так и КМР в целом с учетом влияния препарата на выживаемость [55]. Особую значимость имеет активная медикаментозная профилактика СД2 при наличии предиабета [59] в связи с ограниченной эффективностью мероприятий по изменению образа жизни [60].

При выборе препарата метформина преимущества его формы с пролонгированным высвобождением обусловлены не только снижением побочных эффектов и лучшей переносимостью. Результаты прецедентной paботы Derosa G. et al. (2017) у пациентов с СД2 убедительно демонстрируют большую эффективность метформина с пролонгированным высвобождением в сравнении с обычной формой препарата в отношении улучшения показателей углеводного обмена (гликемии и ее вариабельности, $\mathrm{HbA}_{1 \mathrm{c}}$ ), а также липидного и снижения ИР [61]. Метформин пролонгированной формы высвобождения имеет дополнительное показание - лечение предиабета, кроме того, его можно назначать при клиренсе креатинина $\geq 30$ мл/мин. [62].

\section{ЗАКЛЮЧЕНИЕ}

Дисфункции ЩЖ способствуют различным метаболическим, а также сердечно-сосудистым структурным и функциональным изменениям, повышая КМР. Вследствие высокой распространенности наибольшее внимание привлекает СГипо, который определяют как повышение уровней ТТГ при референсных значениях свободного тироксина и биологически активного трий- одтиронина. Взаимосвязи между субклиническим гипотиреозом и параметрами метаболического синдрома сложные, могут изменяться в зависимости от возраста, пола, индекса массы тела, ИР, курения, потребления йода и маркеров воспаления [63]. Одним из ключевых связующих факторов является именно ИР с ее универсальным характером.

В настоящее время отсутствуют данные о пользе/рисках лечения СГипо препаратами левотироксина, особенно у пациентов старших возрастных групп; не определены пороговые значения ТТГ для инициации приема препарата, отсутствуют данные об улучшении качества жизни и снижении сердечно-сосудистой смертности $[40,42,44,64]$. Влияние метформина, по сути, антидиабетического препарата (по современным представлениям не только антигипергликемического, но и снижающего КМР) на АМФ-киназный сигнальный путь на клеточном уровне предполагает его новый терапевтический потенциал.

В недавнем метаанализе было показано наличие взаимосвязи между уменьшением объема доброкачественных узлов ЩЖ, снижением ИР и уровня ТТГ на фоне терапии метформином, что имеет особое значение с учетом роста заболеваемости предиабетом, СД2 и узловой патологией щЖ [65]. С одной стороны, основным митотическим фактором узлообразования/онкологии ЩЖ является ТТГ, с другой - именно МС и резистентность к инсулину в комплексе с инсулиноподобным фактором роста 1 определяют повышение частоты нарушений углеводного обмена, узлового зоба и папиллярного рака ЩЖ [66].

Возможность коррекции с помощью метформина как периферической, так и центральной ИР, снижения уровней ТТГ при субклиническом гипотиреозе, дополнительном факторе ухудшения сердечно-сосудистого прогноза, подчеркивает перспективность комплексного подхода к профилактике кардиометаболического и, вероятно, онкологического риска.

\section{ДОПОЛНИТЕЛЬНАЯ ИНФОРМАЦИЯ}

Источник финансирования. Выполненная работа не имела финансовой поддержки.

Конфликт интересов. Авторы декларируют отсутствие явных и потенциальных конфликтов интересов, связанных с публикацией настоящей статьи.

Участие авторов. Все авторы внесли значимый вклад в проведение исследования и подготовку статьи, прочли и одобрили финальную версию статьи перед публикацией.

\section{СПИСОК ЛИТЕРАТУРЫ | REFERENCES}

1. Du F-M, Kuang H-Y, Duan B-H, et al. Associations Between Thyroid Hormones Within the Euthyroid Range and Indices of Obesity in Obese Chinese Women of Reproductive Age. Metab Syndr Relat Disord. 2019;17(8):416-422. doi: https://doi.org/10.1089/met.2019.0036

2. Руяткина Л.А., Руяткин Д.С. Интегральный сердечно-сосудистый риск: метаболический синдром и дисфункция щитовидной железы. // Сибирское медицинское обозрение. - 2010. №4. - C. 11-16. [Ruyatkina LA, Ruyatkin DS. Integral cardiovascular risk: metabolic syndrome and thyroid dysfunction. Siberian medical review. 2010;(4):11-16. (In Russ.)]
3. Mohan V, Bodhini D. Mediators of insulin resistance \& cardiometabolic risk: Newer insights. Indian J Med Res. 2018;148(2):127. doi: https://doi.org/10.4103/ijmr.IJMR_969_18

4. Руяткина Л.А., Руяткин Д.С., Исхакова И.С. Возможности и варианты суррогатной оценки инсулинорезистентности. // Ожирение и метаболизм. - 2019. - Т. 16. — №1. - С. 27-32. [Ruyatkina LA, Ruyatkin DS, Iskhakova IS. Opportunities and options for surrogate assessment of insulin resistance. Obesity and metabolism. 2019;16(1):27-32. (In Russ.)] doi: https://doi. org/10.14341/omet10082 
5. Park SY, Park SE, Jung SW, et al. Free triiodothyronine/free thyroxine ratio rather than thyrotropin is more associated with metabolic parameters in healthy euthyroid adult subjects. Clin Endocrinol. 2017;87(1):87-96. doi: https://doi.org/10.1111/cen.13345

6. Yang L, Lv X, Yue F, et al. Subclinical hypothyroidism and the risk of metabolic syndrome: A meta-analysis of observational studies. Endocr Res. 2016;41(2):158-165. doi: https://doi.org/10.3109/07435800.2015.1108332

7. Eftekharzadeh A, Khamseh ME, Farshchi A, Malek M. The Association Between Subclinical Hypothyroidism and Metabolic Syndrome as Defined by the ATP III Criteria. Metab Syndr Relat Disord. 2016;14(3):137-144. doi: https://doi.org/10.1089/met.2015.0065

8. Nakajima Y, Yamada M, Akuzawa M, et al. Subclinical Hypothyroidism and Indices for Metabolic Syndrome in Japanese Women: One-Year Follow-Up Study. J Clin Endocrinol Metab. 2013;98(8):3280-3287. doi: https://doi.org/10.1210/jc.2013-1353

9. Amouzegar A, Mehran L, Takyar M, et al. Tehran Thyroid Study (TTS). Int J Endocrinol Metab. 2018;16(4 Suppl):e84727. doi: https://doi.org/10.5812/ijem.84727

10. Amouzegar A, Kazemian E, Abdi H, et al. Association Between Thyroid Function and Development of Different Obesity Phenotypes in Euthyroid Adults: A Nine-Year Follow-Up. Thyroid. 2018;28(4):458-464. doi: https://doi.org/10.1089/thy.2017.0454

11. Iwen KA, Oelkrug R, Kalscheuer H, Brabant G. Metabolic Syndrome in Thyroid Disease. Front Horm Res. 2018;49:48-66. doi: https://doi.org/10.1159/000485996

12. Razvi S, Jabbar A, Pingitore A, et al. Thyroid Hormones and Cardiovascular Function and Diseases. J Am Coll Cardiol. 2018;71(16):1781-1796. doi: https://doi.org/10.1016/j.jacc.2018.02.045

13. Gyawali P, Takanche JS, Shrestha RK, et al. Pattern of thyroid dysfunction in patients with metabolic syndrome and its relationship with components of metabolic syndrome. Diabetes Metab J. 2015;39(1):66-73. doi: https://doi.org/10.4093/dmj.2015.39.1.66

14. Duntas LH, Chiovato L. Cardiovascular Risk in Patients with Subclinical Hypothyroidism. Eur Endocrinol. 2014;10(2):157-160. doi: https://doi.org/10.17925/EE.2014.10.02.157

15. Biondi B, Cooper DS. The clinical significance of subclinical thyroid dysfunction. Endocr Rev. 2008;29(1):76-131. doi: https://doi.org/10.1210/er.2006-0043

16. Floriani C, Gencer B, Collet TH, Rodondi N. Subclinical thyroid dysfunction and cardiovascular diseases: 2016 update. Eur Heart J. 2018;39(7):503-507. doi: https://doi.org/10.1093/eurheartj/eh×050

17. Mendes D, Alves C, Silverio N, Batel Marques F. Prevalence of Undiagnosed Hypothyroidism in Europe: A Systematic Review and Meta-Analysis. Eur Thyroid J. 2019;8(3):130-143. doi: https://doi.org/10.1159/000499751

18. Tekle HA, Bobe TM, Tufa EG, Solomon FB. Age-sex disparities and subclinical hypothyroidism among patients in Tikur Anbesa Specialized Hospital, Addis Ababa, Ethiopia. J Health Popul Nutr. 2018;37(1):18. doi: https://doi.org/10.1186/s41043-018-0149-x

19. Surks Ml, Ortiz E, Daniels GH, et al. Subclinical thyroid disease: scientific review and guidelines for diagnosis and management. JAMA. 2004;291(2):228-238. doi: https://doi.org/10.1001/jama.291.2.228

20. Polikar R, Burger AG, Scherrer U, Nicod P The thyroid and the heart. Circulation. 1993;87(5):1435-1441. doi: https://doi.org/10.1161/01.cir.87.5.1435

21. Kahaly GJ. Cardiovascular and atherogenic aspects of subclinical hypothyroidism. Thyroid. 2000;10(8):665-679. doi: https://doi.org/10.1089/10507250050137743

22. Tseng FY, Lin WY, Lin CC, et al. Subclinical hypothyroidism is associated with increased risk for all-cause and cardiovascular mortality in adults. J Am Coll Cardiol. 2012;60(8):730-737. doi: https://doi.org/10.1016/j.jacc.2012.03.047

23. Некрасова Т.А., Стронгин Л.Г., Морозова Е.П., и др. Модифицирующее влияние субклинического гипотиреоза на течение артериальной гипертензии: взаимосвязи со скрытой неэффективностью лечения, суточным профилем артериального давления и состоянием органов-мишеней. // Клиническая и экспериментальная тиреоидология. - 2015. - Т. 11. - №2. - C. 55-62. [Nekrasova TA, Strongin LG, Morozova EP, et al. Modifying influence of subclinical hypothyroidism on arterial hypertension: relationship to masked treatment failure, circadian blood pressure profile and target organs status. Clinical and experimental thyroidology. 2015;11(2):55-62. (In Russ.)] doi: https://doi.org/10.14341/ket2015255-62
24. Owen PJ, Sabit R, Lazarus JH. Thyroid disease and vascular function. Thyroid. 2007;17(6):519-524 doi: https://doi.org/10.1089/thy.2007.0051

25. Rodondi N, Bauer DC, Cappola AR, et al. Subclinical thyroid dysfunction, cardiac function, and the risk of heart failure. The Cardiovascular Health study. J Am Coll Cardiol. 2008;52(14):1152-1159. doi: https://doi.org/10.1016/j.jacc.2008.07.009

26. Hak AE, Pols HA, Visser TJ, et al. Subclinical hypothyroidism is an independent risk factor for atherosclerosis and myocardial infarction in elderly women: the Rotterdam Study. Ann Intern Med. 2000;132(4):270-278. doi: https://doi.org/10.7326/0003-4819-132-4-200002150-00004

27. Bielecka-Dabrowa A, Godoy B, Suzuki T, et al. Subclinical hypothyroidism and the development of heart failure: an overview of risk and effects on cardiac function. Clin Res Cardiol. 2019;108(3):225-233. doi: https://doi.org/10.1007/s00392-018-1340-1

28. Dhital R, Poudel DR, Tachamo N, et al. Ischemic Stroke and Impact of Thyroid Profile at Presentation: A Systematic Review and Meta-analysis of Observational Studies. J Stroke Cerebrovasc Dis. 2017;26(12):2926-2934. doi: https://doi.org/10.1016/j.jstrokecerebrovasdis.2017.07.015

29. Gong Y, Ma Y, Ye Z, et al. Thyroid stimulating hormone exhibits the impact on LDLR/LDL-c via up-regulating hepatic PCSK9 expression. Metabolism. 2017;76:32-41. doi: https://doi.org/10.1016/j.metabol.2017.07.006

30. Ebrahimpour A, Vaghari-Tabari M, Qujeq D, et al. Direct correlation between serum homocysteine level and insulin resistance index in patients with subclinical hypothyroidism: Does subclinical hypothyroidism increase the risk of diabetes and cardio vascular disease together? Diabetes Metab Syndr. 2018;12(6):863-867. doi: https://doi.org/10.1016/j.dsx.2018.05.002

31. Sieminska L, Wojciechowska C, Walczak K, et al. Associations between metabolic syndrome, serum thyrotropin, and thyroid antibodies status in postmenopausal women, and the role of interleukin-6. Endokrynol Pol. 2015;66(5):394-403. doi: https://doi.org/10.5603/EP.2015.0049

32. Altay $\mathrm{S}$, Onat $A$, Can $\mathrm{G}$, et al. High-normal thyroid-stimulating hormone in euthyroid subjects is associated with risk of mortality and composite disease endpoint only in women. Arch Med SCi. 2018;14(6):1394-1403. doi: https://doi.org/10.5114/aoms.2016.63264

33. Gupta G, Sharma P, Kumar P, Itagappa M. Study on Subclinical Hypothyroidism and its Association with Various Inflammatory Markers. J Clin Diagn Res. 2015;9(11):BC04-06. doi: https://doi.org/10.7860/JCDR/2015/14640.6806

34. Liu J, Duan Y, Fu J, Wang G. Association Between Thyroid Hormones, Thyroid Antibodies, and Cardiometabolic Factors in Non-Obese Individuals With Normal Thyroid Function. Front Endocrinol (Lausanne). 2018;9:130. doi: https://doi.org/10.3389/fendo.2018.00130

35. Chen Y, Zhu C, Chen Y, et al. Are Thyroid Autoimmune Diseases Associated with Cardiometabolic Risks in a Population with Normal Thyroid-Stimulating Hormone? Mediators Inflamm. 2018;2018:1856137. doi: https://doi.org/10.1155/2018/1856137

36. Roifman I, Beck PL, Anderson TJ, et al. Chronic inflammatory diseases and cardiovascular risk: a systematic review. Can J Cardiol. 2011;27(2):174-182. doi: https://doi.org/10.1016/j.cjca.2010.12.040

37. Xu C, Zhou L, Wu K, et al. Abnormal Glucose Metabolism and Insulin Resistance Are Induced via the IRE1alpha/XBP-1 Pathway in Subclinical Hypothyroidism. Front Endocrinol (Lausanne). 2019;10:303. doi: https://doi.org/10.3389/fendo.2019.00303

38. Brenta G, Caballero AS, Nunes MT. Case Finding for Hypothyroidism Should Include Type 2 Diabetes and Metabolic Syndrome Patients: A Latin American Thyroid Society (Lats) Position Statement. Endocr Pract. 2019;25(1):101-105. doi: https://doi.org/10.4158/EP-2018-0317

39. Chang YC, Hua SC, Chang CH, et al. High TSH Level within Normal Range Is Associated with Obesity, Dyslipidemia, Hypertension, Inflammation, Hypercoagulability, and the Metabolic Syndrome: A Novel Cardiometabolic Marker. J Clin Med. 2019;8(6) doi: https://doi.org/10.3390/jcm8060817

40. Feller M, Snel M, Moutzouri E, et al. Association of Thyroid Hormone Therapy With Quality of Life and Thyroid-Related Symptoms in Patients With Subclinical Hypothyroidism: A Systematic Review and Meta-analysis. JAMA. 2018;320(13):1349-1359. doi: https://doi.org/10.1001/jama.2018.13770 
41. Andersen MN, Olsen AM, Madsen JC, et al. Levothyroxine Substitution in Patients with Subclinical Hypothyroidism and the Risk of Myocardial Infarction and Mortality. PLoS One. 2015;10(6):e0129793. doi: https://doi.org/10.1371/journal.pone.0129793

42. Andersen MN, Olsen AS, Madsen JC, et al. Long-Term Outcome in Levothyroxine Treated Patients With Subclinical Hypothyroidism and Concomitant Heart Disease. J Clin Endocrinol Metab. 2016;101 (11):4170-4177. doi: https://doi.org/10.1210/jc.2016-2226

43. Jasim S, Gharib H. Thyroid and Aging. Endocr Pract. 2018;24(4):369-374 doi: https://doi.org/10.4158/EP171796.RA

44. Leng O, Razvi S. Hypothyroidism in the older population. Thyroid Res. 2019;12:2. doi: https://doi.org/10.1186/s13044-019-0063-3

45. Vigersky RA, Filmore-Nassar A, Glass AR. Thyrotropin suppression by metformin. J Clin Endocrinol Metab. 2006;91(1):225-227. doi: https://doi.org/10.1210/jc.2005-1210

46. Lupoli R, Di Minno A, Tortora A, et al. Effects of treatment with metformin on TSH levels: a meta-analysis of literature studies. J Clin Endocrinol Metab. 2014;99(1):E143-148. doi: https://doi.org/10.1210/jc.2013-2965

47. Fournier JP, Yin $\mathrm{H}, \mathrm{Yu} \mathrm{OH}$, Azoulay L. Metformin and low levels of thyroid-stimulating hormone in patients with type 2 diabetes mellitus. CMAJ. 2014;186(15):1138-1145. doi: https://doi.org/10.1503/cmaj.140688

48. Distiller LA, Polakow ES, Joffe BI. Type 2 diabetes mellitus and hypothyroidism: the possible influence of metformin therapy. Diabet Med. 2014;31(2):172-175. doi: https://doi.org/10.1111/dme.12342

49. Nurcheshmeh Z, Aliasgarzadeh A, Bahrami A, Mobasseri $M$. The Effects of Metformin on Thyroid Function among Patients with Subclinical Hypothyroidism and Coexisting Metabolic Syndrome. Pharm Sci. 2018;24(2):118-123. doi: https://doi.org/10.15171/ps.2018.18

50. Palui R, Sahoo J, Kamalanathan S, et al. Effect of metformin on thyroid function tests in patients with subclinical hypothyroidism: an open-label randomised controlled trial. J Endocrinol Invest. 2019:42(12):1451-1458. doi: https://doi.org/10.1007/s40618-019-01059-w

51. Krysiak R, Szkrobka W, Okopien B. Sex-dependent effect of metformin on hypothalamic-pituitarythyroid axis activity in patients with subclinical hypothyroidism. Pharmacol Rep. 2016;68(6):1115-1119. doi: https://doi.org/10.1016/j.pharep.2016.07.002

52. Meng X, Xu S, Chen G, et al. Metformin and thyroid disease. J Endocrinol. 2017;233(1):R43-R51. doi: https://doi.org/10.1530/JOE-16-0450

53. Cetinkalp S, Simsir IY, Ertek S. Insulin resistance in brain and possible therapeutic approaches. Curr Vasc Pharmacol. 2014:12(4):553-564. doi: https://doi.org/10.2174/1570161112999140206130426

54. Wang B, Cheng KK. Hypothalamic AMPK as a Mediator of Hormonal Regulation of Energy Balance. Int J Mol Sci. 2018;19(11). doi: https://doi.org/10.3390/ijms19113552
55. Руяткина Л.А., Руяткин Д.С. Многоплановые эффекты метформина у пациентов с сахарным диабетом 2 типа. // Сахарный диабет. 2017. - T. 20. - № 3. - C. 210-219. [Ruyatkina LA, Ruyatkin DS. Multidimensional effects of metformin in patients with type 2 diabetes. Diabetes mellitus. 2017;20(2):210-219. (In Russ.)] doi: https://doi.org/10.14341/DM2003458-64

56. Cho K, Chung JY, Cho SK, et al. Antihyperglycemic mechanism of metformin occurs via the AMPK/LXRalpha/POMC pathway. Sci Rep. 2015;5:8145. doi: https://doi.org/10.1038/srep08145

57. Andrade BM, de Carvalho DP. Perspectives of the AMP-activated kinase (AMPK) signalling pathway in thyroid cancer. Biosci Rep. 2014;34(2). doi: https://doi.org/10.1042/BSR20130134

58. Piskovatska V, Stefanyshyn N, Storey KB, et al. Metformin as a geroprotector: experimental and clinical evidence. Biogerontology. 2019;20(1):33-48. doi: https://doi.org/10.1007/s10522-018-9773-5

59. Дедов И.И., Шестакова М.В., Майоров А.Ю., и др. Алгоритмы специализированной медицинской помощи больным сахарным диабетом. / Под ред. Дедова И.И., Шестаковой М.В., Майорова A.Ю. 9-й выпуск. // Сахарный диабет. — 2019. - Т. 22. - №S1. C. 1-144. [Dedov II, Shestakova MV, Mayorov AY, et al. Dedov II, Shestakova MV, Mayorov AY, editors. Standards of specialized diabetes care. 9th ed. Diabetes mellitus. 2019;22(S1):1-144. (In Russ.)] doi: https://doi.org/10.14341/DM221S1

60. Long-term effects of lifestyle intervention or metformin on diabetes development and microvascular complications over 15-year follow-up: the Diabetes Prevention Program Outcomes Study. Lancet Diabetes Endocrinol. 2015;3(11):866-875. doi: https://doi.org/10.1016/s2213-8587(15)00291-0

61. Derosa G, D'Angelo A, Romano D, Maffioli P. Effects of metformin extended release compared to immediate release formula on glycemic control and glycemic variability in patients with type 2 diabetes. Drug Des Devel Ther. 2017;11:1481-1488. doi: https://doi.org/10.2147/dddt.s131670

62. rlsnet.ru [интернет]. Глюкофаж ${ }^{\circledast}$ Лонг (Glucophage ${ }^{\circledast}$ long) [доступ от 25.04.2020]. Доступ по ссылке: https://www.rlsnet.ru/tn_index_id_45613.htm. [Rlsnet.ru [Internet]. Glucophage ${ }^{\circledast}$ long [cited 2020 Apr 25]. Available from: https://www.rlsnet.ru/tn_index_id_45613.htm. (In Russ.)]

63. Mehran L, Amouzegar A, Azizi F. Thyroid disease and the metabolic syndrome. Current Opinion in Endocrinology \& Diabetes and Obesity. 2019;26(5):256-265. doi: https://doi.org/10.1097/med.0000000000000500

64. Chiovato L, Magri F, Carle A. Hypothyroidism in Context: Where We've Been and Where We're Going. Adv Ther. 2019;36(Suppl 2):47-58. doi: https://doi.org/10.1007/s12325-019-01080-8

65. Sui M, Yu Y, Zhang H, et al. Efficacy of Metformin for Benign Thyroid Nodules in Subjects With Insulin Resistance: A Systematic Review and Meta-Analysis. Front Endocrinol (Lausanne). 2018;9:494 doi: https://doi.org/10.3389/fendo.2018.00494

66. Yildirim Simsir I, Cetinkalp S, Kabalak T. Review of Factors Contributing to Nodular Goiter and Thyroid Carcinoma. Med Princ Pract. 2020;29(1):1-5. doi: https://doi.org/10.1159/000503575

\section{ИНФОРМАЦИЯ ОБ АВТОРАХ [AUTHORS INFO]:}

*Руяткина Людмила Александровна, д.М.Н., профессор [Lyudmila A. Ruyatkina, MD, PhD, professor]; ORCID: https://orcid.org/0000-0002-6762-5238; eLibrary SPIN: 1895-7664; e-mail: larut@list.ru

Руяткин Дмитрий Сергеевич, к.м.н. [Dmitriy S. Ruyatkin, MD, PhD]: ORCID: https://orcid.org/0000-0003-3431-5943; eLibrary SPIN: 5433-3153; e-mail: de79@mail.ru

*Автор, ответственный за переписку / Corresponding author.

\section{ЦИТИРОВАТЬ:}

Руяткина Л.А., Руяткин Д.С. Субклинический гипотиреоз и метаболический синдром: основания для медикаментозного вмешательства // Ожирение и метаболизм. - 2020. - Т. 17. - №1. - С. 41-47. doi: https://doi.org/10.14341/omet12282

\section{TO CITE THIS ARTICLE:}

Ruyatkina LA, Ruyatkin DS. Subclinical hypothyroidism and metabolic syndrome: reasons for drug intervention. Obesity and metabolism. 2020;17(1):41-47. doi: https://doi.org/10.14341/omet12282 